\title{
Laser-induced photoconductivity of ferromagnetic semiconductors
}

\author{
O.Yu. Semchuk ${ }^{1}$, R.V. Bila1, M. Willander ${ }^{2}$, M. Karlsteen ${ }^{2}$ \\ ${ }^{1}$ Institute of Surface Chemistry, National Academy of Sciences of Ukraine, 17, General Naumov Street, 03164 Kyiv, Ukraine \\ ${ }^{2}$ Chalmers University of Technology and Goteborg University, SE-41296, Goteborg, Sweden
}

\begin{abstract}
The influence of a strong electromagnetic wave on the kinetic phenomena in ferromagnetic semiconductors (FMSC) is considered. To sequentialy consider this influence, we obtained quantum kinetic equations for electrons and magnons involved into the process of electron-magnon interaction at presence of intense high-frequency electromagnetic field. The new mechanism of a photoconduction not bound with a change of the number of carriers in the conduction band of FMSC and hot conduction electrons is offered. The density of electric current in the FMSC provided by strong variable electromagnetic field and feeble constant electric field has been calculated. The valuation of FMSC electric conductivity in the infrared spectral range is made. It is shown that magnification of the amplitude of variable electromagnetic filed results in increasing the conductivity of FMSC at some fixed temperature.
\end{abstract}

Keywords: photoconductivity, ferromagnetic semiconductor, kinetic phenomena, electromagnetic field, kinetic equation, electron, magnon.

Paper received 12.12.03; accepted for publication 30.03.04.

\section{Introduction}

The strong external electromagnetic field of powerful laser radiation essentially changes kinetics of interacting quasi-particles in semiconductors. This influence, on the one hand, results in a change of energy of quasi-particles during their free run, and, on the other hand, in direct sharing quanta of electromagnetic field - photons in processes of interactions between quasi-particles [1]. This circumstance has also a significant effect on the electrical conductivity of semiconductor being in the field of powerful laser radiation. Laser-induced photoconduction of usual (nonferromagnetic) semiconductors was already investigated earlier [2]. However, in ferromagnetic semiconductors (FMSC) this effect was not explored yet.

In this article, we adduce the result of calculations of the electrical conductivity of FMSC, located in feeble constant electric and arbitrary in its intensity laser radiation external fields. It is shown that the change of the ground state of conduction electrons at the presence of a strong electromagnetic wave results in change of probability of electron-magnon interaction. It, in turn, results in a change of an electrical conductivity of the ferromagnetic semiconductor. We offer a new mechanism of photoconduction not bound with any change of the number of carriers in the conduction band of FMSC and with heating conduction electrons. To simplify calculations, we shall assume that both on energy and impulse electrons relax on magnons. In this electron-magnon interaction, we shall take into account both two-magnon processes in the first order of the perturbation theory, and one-magnon in the second order. It is shown that with increasing the electric strength of the laser wave the magnification of laser-induced photoconduction in FMSC at the same fixed temperature should be observed. It is found that the laser-induced photoconductivity of FMSC is positive and can be enough large in the field of powerful laser radiation.

\section{Features of electron - magnon interaction in FMSC in the field of powerful laser radiation}

Let's consider a wide-band donor FMSC, that is in constant external electric field and external field of powerful laser radiation

$$
\vec{E}(t)=-\frac{1}{c} \frac{\partial \vec{A}(t)}{\partial t}=\frac{\omega}{c} \vec{A} \sin \omega t=\vec{E} \sin \omega t .
$$

To simplify calculations, we shall assume that both on energy and impulse electrons relax on magnons. In this electron-magnon interaction, we shall take into account both two-magnon processes in the first order of the perturbation theory, and one-magnon in the second order. Quantum kinetic equations describing the process of electron-magnon interaction in FMSC for this case can be represented as: 


$$
\begin{aligned}
& \frac{\partial f_{\vec{p}}}{\partial t}+e \vec{F}_{0} \frac{\partial f_{\vec{p}}}{\partial \vec{p}}=\frac{2 \pi}{\hbar} \sum_{p^{\prime}, q^{\prime}, \vec{q}} \sum_{n=-\infty}^{\infty}\left|C_{\vec{p}^{\prime} \vec{q}^{\prime}}\right|^{2} J_{n}^{2}(x) \delta_{\vec{p}+\vec{q}}^{\vec{p}^{\prime}+\vec{q}^{\prime}}\left\{\begin{array}{l}
f_{\vec{p}^{\prime}}\left(1-f_{\vec{p}}\right) N_{\vec{q}^{\prime}}\left(1+N_{\vec{q}}\right)- \\
f_{\vec{p}}\left(1-f_{\vec{p}^{\prime}}\right) N_{\vec{q}}\left(1+N_{\vec{q}^{\prime}}\right)
\end{array}\right\} \delta\left(\Delta_{\left.\vec{p} \vec{p}^{\prime} \vec{q}^{\prime}\right),(2)}\right. \\
& \frac{\partial N_{\vec{q}}}{\partial t}=\frac{2 \pi}{\hbar} \sum_{\vec{p}^{\prime}, \vec{q}^{\prime} n=-\infty} \sum^{\infty}\left|C_{\vec{p}^{\prime} \vec{q} \vec{q}^{\prime}}\right|^{2} J_{n}^{2}(x) \delta_{\vec{p}+\vec{q}+\vec{q}^{\prime}}\left\{\begin{array}{l}
f_{\vec{p}^{\prime}}\left(1-f_{\vec{p}}\right) N_{\vec{q}^{\prime}}\left(1+N_{\vec{q}}\right)- \\
f_{\vec{p}}\left(1-f_{\vec{p}^{\prime}}\right) N_{\vec{q}}\left(1+N_{\vec{q}^{\prime}}\right)
\end{array}\right\} \delta\left(\varepsilon_{\vec{p}^{\prime}}-\varepsilon_{\vec{p}}+\omega_{\vec{q}^{\prime}}-\omega_{\vec{q}}-n \hbar \omega\right),
\end{aligned}
$$

where $J(x)$ is a Bessel function of real argument, $x=\frac{e \vec{E}\left(\vec{P}^{\prime}-\vec{P}\right)}{m \omega^{2}}, C_{\vec{p} \vec{q} r}$ is the matrix element of the electron-magnon interaction including both two-magnon effects in the first order of perturbation theory and also the second-order one-magnon effects, $\Delta_{\vec{p}}^{\vec{p}^{\prime} \vec{q}^{\prime}}=\varepsilon_{\vec{p}^{\prime}}-\varepsilon_{\vec{p}}+\omega_{\vec{q}^{\prime}}-$ $-\omega_{\vec{q}}-n \hbar \omega$.

Before beginning the calculations of FMSC electrical conductivity, it is necessary to make a number of the notes and clarifications. The presence of a high-frequency electromagnetic field, on the one hand, results in a change of an effective matrix element for electronmagnon interaction (occurrence of a factor that contains the Bessel functions $J_{n}$ in integrals of electron-twomagnon and two-magnon-electron interactions), and, on the other hand, in the necessity to update conservation laws (arguments of $\delta$-functions), as now the process of the electron-magnon interaction can be accompanied by absorption of an arbitrary number of photons with the frequency $\omega$. The collision integrals in (2) and (3) do not depend on time obviously. Therefore, there should be a stationary solution of the equations (2) - (3), which features distribution by an electron and magnons in a constant electric field and high-frequency electromagnetic fields averaged by high-frequency oscillations of electrons. The stationary value component current densities in semiconductors under stationary values of high-frequency fields are bound to a stationary component distribution function of electrons $f_{\vec{p}}$, which follows from the solution of the equation (2) as the usual ralation:

$$
\vec{j}=\frac{e}{m} \sum_{\vec{p}} \vec{p} f_{\vec{p}} .
$$

However, to find this solution is not so simply even if to accept the distribution of magnons as equilibrium. The availability of the chosen direction-vector of a high-frequency electric field, results in anisotropy of electronmagnon interaction. In this connection, a sufficiently rigorous solution can be obtained only for the case when the amplitude of high-frequency electric field is not so high and it is possible to be restricted by the square law in its amplitude to the allowances to a distribution function of electrons.

Let's consider the stationary solution of the equation (2), understanding the impulse of an electron as the canonical one $\vec{P}=\vec{p}+\frac{e}{c} \vec{A}(\vec{r}, t)$. Besides, we shall imply that the frequency $\omega$ of intensive laser radiation field satisfies the following inequalities

$\omega>>\tau_{p}^{-1}, \omega<<c / \lambda_{p}, \omega>\omega_{\vec{q}}, \bar{\varepsilon}>>\hbar \tau_{p}^{-1}$.

The first inequality means that the frequency of highfrequency electromagnetic field $\omega$ should be much larger than the reverse relaxation time $\tau_{p}^{-1}$ of electrons by their impulses on magnons under electron-two-magnon interaction. The second inequality means, that the wavelength of an external electromagnetic field $\lambda=c / \omega$ should be much longer than the electron free path. The last inequality demands that the broadening of energy levels of an electron in the course of relaxation should do not exceed the energy value. The above mentioned inequalities enable to be restricted only to significance $n=0$ in Bessel and $\delta$-functions. In a stationary case, the equation (2) is reduced to the following

$$
\begin{aligned}
& e \vec{F}_{0} \frac{\partial f_{\vec{P}}}{\partial \vec{P}}=\frac{2 \pi}{\hbar}\left(\frac{J}{2 N}\right)^{2} \sum_{\vec{q}, \vec{k}} \frac{q^{4}}{\left(q_{0}^{2}+q^{2}\right)^{2}} J_{0}^{2}\left(\frac{e \vec{E} \vec{k}}{m \omega^{2}}\right) \times \\
& \times\left\{\begin{array}{l}
f_{\vec{P}-\vec{k}} N_{\vec{q}+\vec{k}}\left(1+N_{\vec{q}}\right)- \\
f_{\vec{P}} N_{\vec{q}}\left(1+N_{\vec{q}+\vec{k}}\right)
\end{array}\right\} \delta\left(\varepsilon_{\vec{P}-\vec{k}}-\varepsilon_{\vec{P}}+\omega_{\vec{q}}-\omega_{\vec{q}+\vec{k}}\right),
\end{aligned}
$$

where $q_{0}^{2}=2 m J S$.

We shall consider, that the following inequalities take place:

$$
e A<c \sqrt{m \hbar \omega}, \hbar \omega>\bar{\varepsilon} .
$$

It allows us to search for the solution of the equation (5) in dependence from an electric intensity of a field of intensive laser radiation $\vec{E}(t)$ on a perturbation theory. For this purpose we shall present a distribution function of electrons as the following sum

$f_{\vec{P}} \approx f_{0}\left(\varepsilon_{\vec{P}}\right)+\vec{P} \vec{E} f_{1}(\vec{P})$

In $f_{0}\left(\varepsilon_{\vec{P}}\right)$ conditions considered by us, the function has the Maxwell form:

$$
f_{0}\left(\varepsilon_{\vec{P}}\right)=\frac{n}{(2 \pi m T)^{\frac{3}{2}}} e^{-\frac{P^{2}}{2 m}} .
$$

In zero approximation, the function $f_{1}(\vec{P})$ can be found as a solution of the equation (5) in the homogeneous external electric field $\vec{F}_{0}$ (for $\vec{A}=0$ ) and, as it is usual, in diffusion approach (in the relaxation time approximation). For a presence of a relaxation time, we shall make 
the following. At first, for facilitation of accounts, we shall average the quantity $J_{0}^{2}\left(\frac{e \vec{E} \vec{k}}{m \omega^{2}}\right)$ by all the directions of the vector $\vec{k}$ and in (5) we shall make replacement

$$
\begin{aligned}
& J_{0}^{2}\left(\frac{e \vec{E} \vec{k}}{m \omega^{2}}\right) \rightarrow \frac{1}{4 \pi} \int_{0}^{2 \pi} \int_{0}^{\pi} J_{0}^{2}\left(\frac{e E k \cos \theta}{m \omega^{2}}\right) \sin \theta d \theta d \varphi= \\
& =\int_{0}^{1} J_{0}^{2}\left(\frac{e E k x}{m \omega^{2}}\right) d x .
\end{aligned}
$$

Now using the formula

$$
\sum_{\vec{k}} F(\vec{k})=\frac{V}{(2 \pi \hbar)^{3}} \iiint_{3 . \sigma .} F(\vec{k}) d^{3} k .
$$

Let's pass from summation on $\vec{q}$ and $\vec{k}$ to integration over the Brillouin zone. According to that, the equation (5) now can be copied in the following manner:

$$
\begin{aligned}
& e \vec{F}_{0} \frac{\partial f_{\vec{P}}}{\partial \vec{P}}=\frac{2 \pi}{\hbar}\left(\frac{J}{2 N}\right)^{2} \frac{V^{2}}{(2 \pi \hbar)^{6}} \times \\
& \times \int_{3 . \sigma} d \vec{q} d \vec{k} \frac{q^{4}}{\left(q_{0}^{2}+q^{2}\right)^{2}} \int_{0}^{1} J_{0}^{2}\left(\frac{e E k x}{m \omega^{2}}\right) d x \times \\
& \times\left\{\begin{array}{l}
f_{\vec{P}-\vec{k}} N_{\vec{q}+\vec{k}}\left(1+N_{\vec{q}}\right)- \\
f_{\vec{P}} N_{\vec{q}}\left(1+N_{\vec{q}+\vec{k}}\right)
\end{array}\right\} \delta\left(\begin{array}{l}
\varepsilon_{\vec{P}-\vec{k}}-\varepsilon_{\vec{P}}+ \\
\omega_{\vec{q}}-\omega_{\vec{q}+\vec{k}}
\end{array}\right),
\end{aligned}
$$

Representing now the electron distribution function as (6) of (10) we have:

$$
e \vec{F}_{0} \frac{\partial f_{0}\left(\varepsilon_{\vec{P}}\right)}{\partial \vec{P}}=-\frac{1}{\tau_{P}^{e m}(P)}\left(\vec{P} \vec{F}_{0}\right) f_{1}\left(\varepsilon_{\vec{P}}\right)
$$

Here, the quantity $\tau_{p}^{e m}(P)$ is the relaxation time of electrons by their impulse on magnons, which is bound to the influence of powerful laser radiation field. When calculating $\tau_{p}^{e m}(P)$ we shall start from the equation (10). With this aim, we use the energy conservation law according to which

$$
f_{1}\left(\varepsilon_{\vec{P}-\vec{k}}\right)=f_{1}\left(\varepsilon_{\vec{P}}+\omega_{\vec{q}+\vec{k}}-\omega_{\vec{q}}\right) \text {. }
$$

In approach of a relaxation time, including oscillations of a spin subsystem of FMSC low-frequency with (12), it follows that $f_{1}\left(\varepsilon_{\vec{P}-\vec{k}}\right) \approx f_{1}\left(\varepsilon_{\vec{P}}\right)$. Also noteworthy is that from the energy conservation law for electron-twomagnon scattering it follows, that the impulse, transferred in electron scattering $\vec{k}$ should be changed within limits $0<\vec{k}<2 \vec{P}$, and impulse of a magnon - within the limits of the Brillouin zone $\left(0<\vec{q}<\frac{\pi \hbar}{a}\right)$. Fulfilling integra- tion in (10) for an OX- axis, we shall choose a direction of the vector $\vec{F}_{0}$ and enter angles $\vartheta$ and $\alpha$ between vectors $\vec{P}$ both $\vec{F}_{0}$ and $\vec{k}$ and $\vec{F}_{0}$, accordingly. In a Fig. 1 the vectors $\vec{P}, \vec{k}$ and $\vec{F}_{0}$ and angles between them are submitted.

The angles are bound by the following relation:

$\cos \alpha=\cos \vartheta \cos \theta+\sin \vartheta \sin \theta \cos \Phi$,

where $\theta$ and $\Phi$ - polar and azimuth angles, which determine a direction of a vector $\vec{k}$.

Then, we can write the usual expression for the relaxation time of electrons by their impulse when scattering on magnons $\tau_{p}^{e m}(P)$ at the presence of high-frequency electromagnetic field

$\frac{1}{\tau_{p}^{e m}(P)}=\frac{1}{\tau_{p}^{e m}(\varepsilon)} \Psi\left(\frac{2 e E P}{m \omega^{2}}\right)$

where

$\Psi\left(\frac{2 e E P}{m \omega^{2}}\right)=\int_{0}^{1}\left\{J_{0}^{2}\left(\frac{2 e E P}{m \omega^{2}} x\right)+\frac{2}{5} J_{1}^{2}\left(\frac{2 e E P}{m \omega^{2}} x\right)\right\} d x$.

For a sufficiently small amplitude of this field or for its very major frequency $\omega$, it is received $z=\frac{2 e E P}{m \omega^{2}} \ll 1$, therefore $\Psi(z)=1$. Hence, in this case

$\tau_{p}^{e m}(P) \approx \tau_{e m}^{p}(\varepsilon)=\frac{8 \pi^{2} \hbar^{7} \sqrt{J S}}{a^{6} m_{s}^{2} m J^{2} \Theta_{m} \sqrt{\varepsilon}}$

$\left(\tau_{e m}^{p}(\varepsilon)\right.$ is the electron relaxation time by the impulse on magnons in the absence of powerful laser radiation [5] and influence of this field on the process of electronmagnon interaction will be incidental. More interesting will be a case when the amplitude of the electric field of the laser wave will be major when $z>>1$. Using the known asymptotic series of the Bessel function for this case

$J_{n}(z) \approx \sqrt{\frac{2}{\pi z}} \cos \left(z-\frac{\pi}{2} n-\frac{\pi}{4}\right)$.

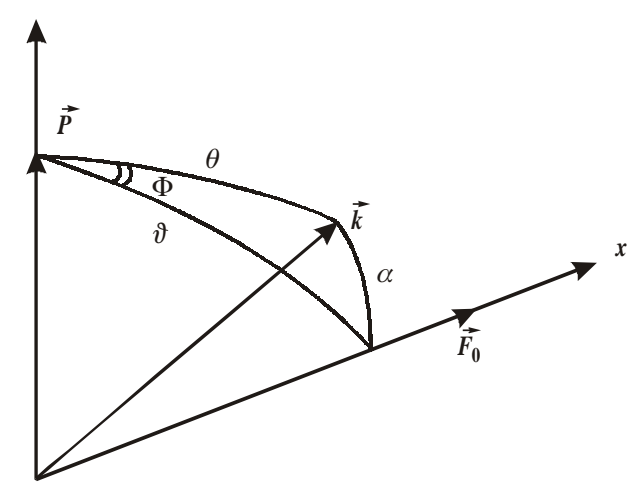

Fig. 1. Mutual orientation of vectors $\vec{P}, \vec{k}$ and $\vec{F}_{0}$ in space. 
and substituting (16) in (14) for want of $n=0$, we receive $\Psi(z) \approx \frac{1}{\pi z} \ln z$.

Hence, in this case for the relaxation time of electrons by their impulse when scattering on magnons $\tau_{p}^{e m}(P)$ at the presence of the laser wave field, we have the following expression:

$\tau_{p}^{e m}(P)=\tau_{p}^{e m}(\varepsilon) \frac{2 \pi|e| E P}{m \omega^{2}}\left[\ln \frac{2 \pi|e| E P}{m \omega^{2}}\right]^{-1}$.

Hence, for the major amplitude of of the laser wave electric field, the relaxation time of electrons on an impulse on magnons becomes function of frequency and amplitude of a field of a laser wave.

\section{Density of an electrical current in FMSC in a powerful field of laser radiation}

The density of electrical current in FMSC being in the field of a laser wave (1) and feeble constant electric fields $\vec{F}_{0}$ is determined by the formula (4). As the summation in (4) happens on all the range of impulses, the choice of a distribution function for electrons $f_{\vec{p}}$ in the representation of canonical impulse or in the gradient-invariant form is equivalent. It means that substitution of decompositions (6) into (4) owing to symmetry of the function $f_{0}\left(\varepsilon_{\vec{p}}\right)$ concerning replacement $\vec{P}$ on $-\vec{P}$ does not give a contribution into the electrical conductivity. Therefore, for an electrical conductivity we shall have now the following expression

$j=\frac{2 e}{m} \sum_{\vec{P}} \vec{P}\left(\vec{P} \vec{F}_{0}\right) f_{1}\left(\varepsilon_{\vec{P}}\right)$.

Let's note that the factor 2 in (18) takes into account summation after both projections of a spin of an electron. In approach of a relaxation time, the function $f_{1}\left(\varepsilon_{\vec{P}}\right)$ follows directly from the equation (11). As

$$
\frac{\partial f_{0}\left(\varepsilon_{\vec{P}}\right)}{\partial \vec{P}}=\frac{\vec{P}}{m} \frac{\partial f_{0}\left(\varepsilon_{\vec{P}}\right)}{\partial \varepsilon_{\vec{P}}},
$$

that from (11) one can receive

$$
f_{1}\left(\varepsilon_{\vec{P}}\right)=-\frac{e}{m} \tau_{p}^{e m}(P) \frac{\partial f_{0}\left(\varepsilon_{\vec{P}}\right)}{\partial \varepsilon_{\vec{P}}} .
$$

Substituting (20) into (18) and executing integration, for $i$-th $(i=x, y, z)$ component of a current density caused by influence of a laser wave, we receive the following relation:

$$
j_{E}^{i}=-\frac{2 e^{2}}{m^{2}} \frac{V}{(2 \pi \hbar)^{3}} \int d \vec{P} P_{i}\left(\vec{P} \vec{F}_{0}\right) \tau_{p}^{e m}(P) \frac{\partial f_{0}\left(\varepsilon_{\vec{P}}\right)}{\partial \varepsilon_{\vec{P}}} .
$$

From (21), we receive the expression for the current density $j_{E}$ along the direction of the constant electric field $\vec{F}_{0}$

SQO, 7(1), 2004
$j_{E}=j_{0} \Phi(\alpha)$,

where $j_{0}$ is the current density in FMSC in absence of a field of powerful laser radiation [5], and $\Phi(\alpha)$ is the function, that describes the influence of intens laser radiation on the electrical conductivity of FMSC, where

$\alpha=\frac{|e| E}{\omega^{2}} \sqrt{\frac{8 T}{m}}$

is the parameter, that depends on an electric intensity of a laser wave.

For major and small amplitudes of the laser wave intensity, the function $\Phi(\alpha)$ has two various asymptotics:

$$
\Phi(\alpha)= \begin{cases}1+\frac{1}{9} \alpha^{2}, & \alpha<<1, \\ \frac{9 \pi^{\frac{3}{2}}}{16} \frac{\alpha}{\ln \alpha}, & \alpha>>1 .\end{cases}
$$

Thus, the stationary value component of electric current density $j$ in FMSC in the field of the laser radiation can be represented in the following form:

$j=j_{0}+j_{E}=j_{0}+j_{0} \Phi(\alpha)$.

From here we received the following expression for a laser-induced photoconductivity $j_{p h}=j-j_{0}$

$$
j_{p h}=j_{0} \Phi(\alpha) \text {. }
$$

Hence, both for major and small values of $a$ from (26) follows that with increasing the electric intensity of a laser wave $E$ (according to (23) the increase of the laser wave amplitude results in increase of the parameter $a$ ) the increase in photoconductivity at the same fixed temperature should be observed. Above told is illustrated by a pictorial dependence of the dimensionless photoelectric current $j_{p h} / j_{0}$ on the dimensionless electric field of the laser wave $x=\frac{E}{E_{0}}\left(E_{0}=\frac{\omega^{2}}{e} \sqrt{\frac{m}{8 T}}\right)$, in Figs 2 and 3 .

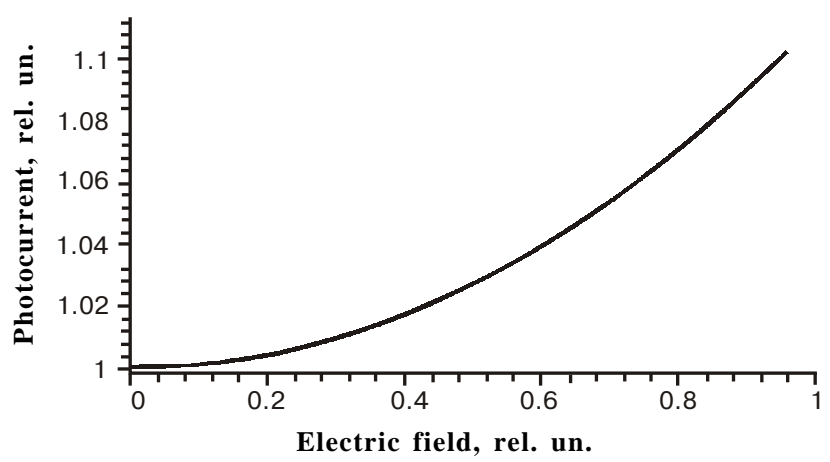

Fig. 2. Dependence of a photoelectric current on an electric intensity of a laser wave $(0<x<1)$. 
O.Yu. Semchuk et al.: Laser-induced photoconductivity of ferromagnetic semiconductors

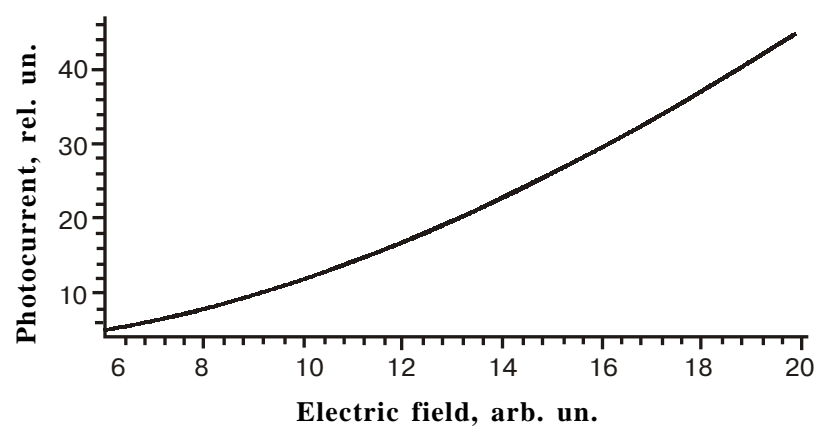

Fig. 3. Dependence of a photoelectric current on an electric intensity of a laser wave $(x>0)$.

Thus, the laser-induced photoconductivity in FMSC is a positive quantity and can essentially increase in the field of powerful laser radiation.

The procedure of calculation of the laser-induced photoconductivity in FMSC, above told, may be used also for examination of relaxation processes in metals, however, for want of it is necessary to take into account that in metals the conduction electrons have impulses close to impulses $p_{F}$, inherent to electrons at the Fermi surface.

\section{References}

1. V.P. Seminozhenko, Kinetics of interacting guasiparticles under strong external fields // Phys. Rep., 91, 104-184 (1982).

2. E.M. Epstein, Scattering electrons by phonons in a strong radiation field // Sov. Phys. Solid State, 11, 2732-2738 (1969),

3. O.Yu. Semchuk., A.E. Levshin., P.M. Tomchuk, Superlattices, formed in ferromagnetic semiconductors by coherent light beams // Sov. Phys. Solid Statev., 28, 229-232 (1986).

4. A.Yu. Semchuk, L.G. Grechko, V.M. Ogenko, Field influence on the parameters of ferromagnetic semiconductor superlattices formed by coherent light beams // Phys. stat. sol. (b), 157, pp. 451-458 (1990).

5. O.Yu. Semchuk., S.S Rozhkov, Heating of electrons and magnons in ferromagnetic semiconductor // Sov. Phys. Solid State, 23, pp. 1118-1120 (1981).

6. G. Korn, T. Korn, Mathematical handbook for scientists and engineers, Mc Grav-Hilll Book Company, INC, New York, Toronto, London, 1961. 